\title{
Editorial
}

\section{Towards gene therapy for cystic fibrosis: a clinical progress report}

A combination of factors have contributed to cystic fibrosis (CF) being one of the pathfinders for clinical studies of gene transfer. The gene for this monogenic disorder was cloned in 1989 and at least one function of the cystic fibrosis transmembrane conductance regulator (CFTR) protein, as a cAMP-activated chloride channel, has been clearly established, providing a convenient marker for gene transfer in vivo. The principal target cells are those of the respiratory epithelium, likely targetable by topical application. Further, CF is a common disorder allowing clinical studies to be carried out at a number of centres. Finally, although conventional treatment has increased life expectancy to approximately 30 years, there is clearly an urgent need for a novel therapeutic approach.

Progress towards clinical studies quickly followed cloning of the CFTR gene. Within a year, two reports demonstrated in vitro correction of the chloride channel defect following gene transfer. Studies in CF transgenic mice followed 3 years later, and showed that liposomemediated gene delivery to the airways could also achieve this in vivo. Subsequently, over the past 4 years a number of groups in the USA and Europe have initiated clinical studies of gene transfer in CF subjects. Three classes of gene transfer agent have been used, adenovirus, cationic lipid and most recently adeno-associated virus. Most studies have used the nasal epithelium as a model for the eventual target organ, the lung, although the latter has also been addressed. What progress has been made?

The first human trial involved the use of adenoviruses, ${ }^{1}$ since when six further studies have been initiated using these agents, with four published..$^{2-5}$ Of the 30 noses assessed following single application, approximately onethird showed some evidence of functional changes in chloride transport, with very few side-effects. However, one trial ${ }^{3}$ reported ipsilateral inflammation that limited the adenoviral dose that could be administered. Following repeated application, ${ }^{5}$ the level of correction of the chloride defect reduced, probably related to immune mechanisms. Data for 10 lungs have also been reported. The initial finding ${ }^{2}$ of an inflammatory response in one subject has not been observed since the method of administration was switched from direct instillation to nebulisation. No functional data were assessed in these pulmonary studies, although three of the subjects showed evidence for either CFTR mRNA or protein. Adeno-associated viruses have been used in three studies, one in the nasal epithelium and two by direct instillation into the nasal sinuses. The former is ongoing in a dose-escalation approach. Preliminary reports from the phase I sinus study indicate some correction of the chloride defect, associated with the long-term presence of vector DNA, whilst a phase II study to reduce the incidence of clinical sinusitis is also underway. There have been no reports of toxicity.

Data for cationic lipids have now been reported from four studies which focused on the nasal epithelium. The first, $^{6}$ in 15 subjects, used the lipid DC-Cholesterol and demonstrated transient correction of the chloride defect in the noses of approximately one-third of treated subjects, without any safety problems. Two recent reports on nasal application in this Journal have further developed these findings. The Edinburgh group ${ }^{7}$ used a different CFTR plasmid (CMV rather than SV40 promoter) and a different lipid (DOTAP). Transgene DNA could be detected up to 28 days following administration in the majority of patients, and two of eight subjects showed some degree of correction of the chloride defect. The study by the Oxford-Cambridge team ${ }^{8}$ used an RSVdriven CFTR plasmid complexed with DC-Cholesterol, with evidence for CFTR function documented in six of eight treated subjects, in some cases into the non-CF range. Neither study noted any safety problems. A further study has compared the application of a DNAlipid (Genzyme lipid 67) complex to one nostril with DNA alone, to the other. ${ }^{9}$ Both were capable of producing vector-specific transcripts and partially correcting the chloride defect. Overall in the nasal epithelium there is a very similar pattern to that found with adenoviral application, with approximately one-third of treated subjects showing some degree of bioelectrical correction. No important toxicity problems were observed or limited the dose administration. Finally, a study of multiple application to the nasal epithelium has recently been completed by the Oxford-Cambridge consortium, demonstrating evidence for CFTR gene transfer in approximately $50 \%$ of the treated patients after each of three applications.

In addition to the nasal studies, the Brompton group has recently completed a study of administration to the lungs in 16 subjects using the Genzyme lipid 67. Measurements of chloride secretion in the lower airways showed evidence for correction of the basic CF defect in the majority of subjects with an average level of approximately $25 \%$ of non-CF values. Each of the DNA-lipidtreated patients, but not the lipid-treated controls, showed a mild flu-like response over a period of approximately $24 \mathrm{~h}$ which did not require specific treatment. One possible explanation for this response may relate to a recent study ${ }^{10}$ showing that DNA of bacterial origin, 
which does not show cytosine methylation of CpG dinucleotides, produces inflammation when instilled into animals' lungs. Administration of either mammalian DNA, which is typically methylated at CpG dinucleotides, or premethylated bacterial DNA did not induce this response.

In summary, within 8 years of the cloning of the CFTR gene, proof-of-principle for correction of the basic defect has been achieved within the target organ in vivo in $\mathrm{CF}$ subjects. Each of the three gene transfer agents used has achieved limited success, with no one outshining the others. Adenoviruses are clearly capable of inducing marked pulmonary toxicity, but with respect to clinical studies an efficacy-toxicity window is being established. However, the reduced efficacy on repeated application, demonstrated both in animal and human studies, and likely related in part to neutralising antibodies, remains an important caveat. Lipids remain less efficient than adenoviruses in laboratory and animal studies, although not demonstrably so in man. The emphasis is now on an understanding of the biological barriers to gene transfer for both of these agents. Adeno-associated viruses are relative newcomers to the clinical arena, and further data are awaited.

Whilst this is encouraging, it is clear that there are many important hurdles still to overcome. Gene transfer efficiency likely needs to be increased in vivo in man. Whilst important progress is being made in improving gene transfer agents, a further focus noted above, is the native biological barriers. The mucosal surface of the respiratory epithelium is primarily a protective layer, not given to uptake of macromolecules. Added to this is the presence of normal mucus, and in the case of CF subjects, a discontinuous barrier of purulent secretions. Each of these, as well as the surface glycocalyx, has been shown to represent important impediments to gene transfer. Even once inside the cell, the majority of gene transfer agents lead to uptake into endosomes, not conducive (particularly for plasmid DNA) to efficient transport to the nucleus. Finally, even if release from the endosome can be effected, transport to and through the nuclear pore complex must be achieved. A recent demonstration of cytoplasmic endonucleases, which digest the plasmid DNA, only add further hazard to an already difficult journey.

Each of the gene transfer systems noted above results only in episomal maintenance of the vector DNA, likely in part to contribute to the limited expression profile of 1-2 weeks seen in man. Whilst this lack of genomic integration is to be welcomed for safety reasons, it brings with it the need for repeated administration. As noted above for adenoviruses this is proving problematic. Lipids have not run into this problem to date, but the toxicological effect of multiple applications of lipids to the human lung is uncertain and needs to be addressed.

As studies progress towards phase III and potential clinical benefit, it is clear that we need assays which bridge the gap between basic bioelectric measurements and clinical improvement. Measurements of pulmonary inflammation and bacterial burden are likely key players, and have been developed for monitoring of other chronic lung diseases. Finally, the choice of subjects for clinical trials is increasingly coming into focus. The earlier in disease progression the better, but this may bring with it the need for long-term follow-up to detect convincing clinical benefit. More severe disease may be refractory to gene transfer because of the increasingly adverse biological barriers, as well as providing an increasingly damaged target. Paediatric gene transfer is clearly an important area to develop, but brings new ethical and toxicological problems, as well as the above noted difficulties of markers of successful gene transfer.

Conventional drugs usually take a decade to move from laboratory to clinic; CF gene therapy is well on course.

EWFW Alton ${ }^{1,6}$, DM Geddes ${ }^{1}$, DR Gill ${ }^{2}$, CF Higgins ${ }^{2,3}$, SC Hyde ${ }^{2}$, JA Innes ${ }^{4}$ and DJ Porteous ${ }^{5,6}$

${ }^{1}$ Ion Transport Unit, Imperial College at the National Heart and Lung Institute, Manresa Road, London SW3 6LR; ${ }^{2}$ Nuffield Department of Clinical Biochemistry and Imperial Cancer Research Fund Laboratories, John Radcliffe Hospital, Oxford; ${ }^{3} \mathrm{MRC}$ Clinical Sciences Centre, Imperial College School of Medicine, Hammersmith Hospital, London, ${ }^{4}$ Department of Respiratory Medicine; and ${ }^{5}$ Medical Research Council Human Genetics Unit, Western General Hospital, Edinburgh, UK;

${ }^{6}$ Authors appear in alphabetical order

\section{References}

1 Zabner $\mathrm{J}$ et al. Adenovirus-mediated gene transfer transiently corrects the chloride transport defect in nasal epithelia of patients with CF. Cell 1993; 75: 207-216.

2 Crystal RG et al. Administration of an adenovirus containing the human CFTR cDNA to the respiratory tract of individuals with cystic fibrosis. Nat Genet 1994; 8: 42-51.

3 Knowles MR et al. A controlled study of adenoviral-vectormediated gene transfer in the nasal epithelium of patients with cystic fibrosis. New Engl J Med 1995; 333: 823-831.

4 Bellon $\mathrm{G}$ et al. Aerosol administration of a recombinant adenovirus expressing CFTR to cystic fibrosis patients: a phase I clinical trial. Hum Gene Ther 1997; 8: 15-25.

5 Zabner $\mathrm{J}$ et al. Repeat administration of an adenovirus vector encoding cystic fibrosis transmembrane conductance regulator to the nasal epithelium of patients with cystic fibrosis. J Clin Invest 1996; 97: 1504-1511.

6 Caplen NJ et al. Liposome-mediated CFTR gene transfer to the nasal epithelium of patients with cystic fibrosis. Nature Med 1995; 1: 39-46.

7 Porteous DJ et al. Evidence for safety and efficacy of DOTAP cationic liposome-mediated CFTR gene transfer to the nasal epithelium of patients with cystic fibrosis. Gene Therapy 1997; 4 : 210-218.

8 Gill DR et al. A placebo-controlled study of liposome-mediated gene transfer to the nasal epithelium of patients with cystic fibrosis. Gene Therapy 1997; 4: 199-209.

9 Zabner J et al. Comparison of DNA-lipid complexes and DNA alone for gene transfer to cystic fibrosis airway epithelia in vivo. J Clin Invest 1997; 100: 1529-1537.

10 Schwartz DA, Quinn T, Thorne PS. CpG motifs in bacterial DNA cause inflammation in the lower respiratory tract. J Clin Invest 1997; 100: 68-73. 